\title{
CARBON MONOXIDE AND THE TEMPERATURE STRUCTURE OF THE SOLAR ATMOSPHERE
}

\author{
T.R. AYRES \\ Center for Astrophysics and Space Astronomy \\ University of Colorado, Boulder, CO 80309 USA
}

\begin{abstract}
The themes woven into this review are: (1) the solar outer atmosphere is highly inhomogeneous; (2) anomalies in the behavior of particular wavelength intervals can signal key inconsistencies in an accepted model; and (3) the observations still hold surprises, particularly in the thermal infrared.
\end{abstract}

\section{Introduction}

The surface layers of the Sun serve as a crucial boundary condition for processes of the deep interior; for example, the reflector for trapped acoustic modes, undeniably relevant to the present Symposium. The stratification of the solar atmosphere once was thought to be well understood, based on a wealth of ultraviolet and optical diagnostics (Vernazza, Avrett, \& Loeser 1973).

Over the past two decades, however, studies of infrared molecular spectra have unearthed puzzling anomalies. Strong lines of the $\Delta v=1$ (fundamental) vibration-rotation bands of carbon monoxide near $4.7 \mu \mathrm{m}$ display very cool temperatures at the extreme limb (Noyes \& Hall 1972), and remarkable off-limb emissions protruding hundreds of kilometers into the supposedly hot chromosphere (Solanki, Livingston, \& Ayres 1994). One proposal is that the low chromosphere is not hot at all, but instead is permeated by CO-cooled gas (Ayres 1981) - a "COmosphere" (Wiedemann et al. 1995), if you will.

The conflicting pictures of hot chromosphere versus cold COmosphere have raised suspicions that those "layers" of the atmosphere are considerably more inhomogeneous than previously suspected. Here, I will describe the infrared molecular evidence in favor of the COmosphere, drawing mainly on recent work at the National Solar Observatory's McMath-Pierce telescope.

I will begin at the end. After briefly describing the classical picture, I will present the punchline; a modern view of the solar atmosphere based on a 


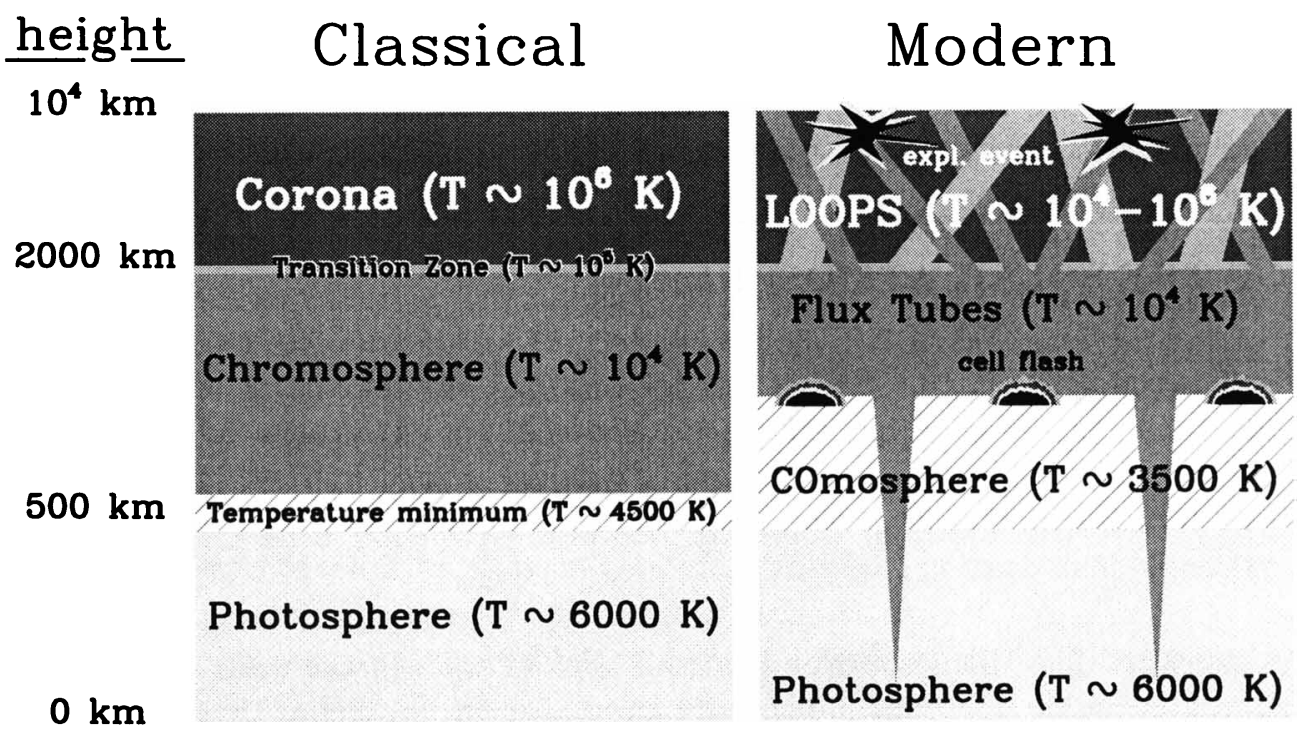

Figure 1. Classical and modern views of solar atmosphere. Height " 0 " is at $\tau_{5000}=1$.

synthesis of ultraviolet and infrared diagnostics. I then will focus on aspects of the model which are illuminated by infrared work, and discuss areas of controversy. The ultraviolet side of the story is just as important. Timely discussions can be found in recent reviews by Avrett (1997) and Ayres (1997).

\section{Atmospheric Paradigms}

\subsection{THE CLASSICAL VIEW: LAYERS}

The classical model, illustrated in the lefthand panel of Figure 1, is a planeparallel stratification. The outer atmosphere is divided into a hierarchy of distinct layers, controlled by different energy budgets, and through which the temperature runs smoothly. The layered atmosphere represents the idealized response of a hydrostatically stratified gas in which the mechanical heating varies slowly with height. The lower portion of the model is the photosphere, where the energy flow is dominated in the deepest levels by convection and in the higher levels by radiation. As the density falls with altitude, the radiative equilibrium becomes increasingly disrupted by mechanical heating. At a critical height the outwardly falling temperature profile bottoms out and begins to rise. That temperature minimum marks the interface between the radiative-heating dominated lower atmosphere, and the mechanical-heating dominated higher layers. 
The temperature continues rising slowly through a region, many scale heights thick, deemed the chromosphere. There, the progressive ionization of hydrogen - with its large reservoir of bound electrons - can fuel the collisionallyexcited line cooling side of the energy budget, roughly independent of the outwardly plummeting hydrogen density. After the pressure has fallen several orders of magnitude through the extensive chromospheric temperature "plateau," the hydrogen is mostly ionized, the reservoir of bound electrons depleted, and the gas no longer can maintain a high $n_{\mathrm{e}}$-and the associated radiative cooling. A thermal instability ensues, and the gas is driven to coronal temperatures $\left(T \sim 10^{6} \mathrm{~K}\right)$ through a thin transition zone (TZ).

The cooling of the coronal layers is accomplished partly by thermal conduction back to the chromosphere and partly by high-temperature optically-thin line and continuum emission (Athay 1976). In this picture, the location of the second key interface, the TZ, is determined by the overall level of heating. The TZ will occur at relatively low altitudes (high densities) if the mechanical heating is large (as in active regions), and at higher altitudes if the heating is less (as in the quiet Sun).

Within the context of the simple layered view it is possible to derive scaling laws that connect, for example, the thickness of the chromospheric plateau and its mean electron density, with stellar surface gravity and the average level of nonradiative heating (Ayres 1979). Furthermore, the layered view naturally is compatible with $1 \mathrm{D}$ numerical simulations of line and continuum formation. Sophisticated NLTE spectral synthesis algorithms have been devised to infer optimum plane-parallel thermal profiles from a wide range of multiwavelength solar data (Vernazza, Avrett, \& Loeser 1973), including the possibility of treating horizontal spatial inhomogeneities by distributions of mildly perturbed $1 \mathrm{D}$ models (Vernazza, Avrett, \& Loeser 1981; Fontenla, Avrett, \& Loeser 1993). The layered $1 \mathrm{D}$ paradigm also is a natural starting point for investigations of late-type coronal stars, lacking all the fine detail of routine solar observations.

\subsection{THE MODERN PICTURE: STRUCTURES}

The new paradigm, illustrated on the righthand side of Fig. 1, is not particularly new at all, but has been around in one form or another ever since the first high spatial resolution pictures of the chromosphere were taken decades ago. The lower part - the photosphere-is identical to that of the layered model. In the middle photosphere, above the convective overshoot region, the horizontal variation in temperature probably is rather mild compared with that at higher altitudes, and any inhomogeneities can be treated successfully by a perturbed standard model. The large departures between the classical and modern views are confined to the heights above the photosphere, dominated by structures.

For example, the fact that the corona exists not as a layer of uniform tem- 
perature and density, but rather as a collection of distinct loop-like structures, dates back at least to the Skylab era (e.g., Vaiana \& Rosner 1978). Contemporary daily images from $S O H O$ EIT and Yohkoh SXT have reinforced the view, emphasizing the wide range of temperatures and densities in coronal magnetic structures, particularly in active regions (Gurman 1997).

We now think of the "transition zone" not only in terms of conductive interfaces at the footpoints of hot coronal loops, but also as a separate population of usually small-scale "warm" $\left(T \sim 10^{5} \mathrm{~K}\right)$ structures mostly associated with the chromospheric network, although larger-scale features occasionally are seen, such as "plumes" over sunspots (Foukal 1975). Even cooler structures $\left(T \sim 10^{4} \mathrm{~K}\right)$ exist in the "corona:" prominences and spicules (Athay 1976; and more recently-Hirayama 1985; Suematsu, Wang, \& Zirin 1995).

The jumble of magnetic fields which constitutes the corona is rooted in a canopy (e.g., Solanki \& Steiner 1990), occupying what previously we would have called the upper chromosphere. The canopy itself is further rooted mainly in the network, by slender funnel-shaped flux tubes that penetrate down through the photosphere into the convection zone, ultimately connecting to the deep source region of the magnetic flux. Interactions between the tangled fields in and above the canopy can give rise to localized explosions traced by high-velocity jets in TZ-temperature lines (the so-called TZ explosive events; e.g., Dere, Bartoe, \& Brueckner 1989; and more recently-Moses et al. 1994; Innes et al. 1997; cf., the nanoflares of Parker 1983).

The pervasive canopy zone in the middle chromosphere is threaded by warm gas with large horizontal density contrasts. Some of the "chromospheric" emission we normally associate with that zone in the layered view arises in the canopy. Additional emission comes from the subcanopy extensions of the flux tubes, which evidently are hotter than the surrounding "quiet" medium down into the middle photosphere. A third component of chromospheric emission arises from cell flashes at the base of the canopy (the acoustic disturbances described by M. Carlsson at this Symposium; see, also, Carlsson \& Stein 1995, 1997; Carlsson, Judge, \& Wilhelm 1997). The canopy emission is ubiquitous and its density striations are traced in $\mathrm{H} \alpha$ filtergrams (e.g., Zirin 1988). The cell flash and low-altitude flux tube components are compact, giving rise to transient and persistent bright points in, for example, Ca K and CN spectroheliograms (Sheeley 1969).

Between the upper photosphere $(h \sim 500 \mathrm{~km})$ and the base of the canopy $(h \sim 1000 \mathrm{~km}$ ) lies a region of controversy, the COmosphere mentioned previously. For reasons that I will describe in more detail later, the cool zone likely is a pervasive component of the outer atmosphere, replacing what previously was designated the low chromosphere. The "cold clouds" surrounding the hot flux tubes probably owe their existence to strong radiative cooling by the $\mathrm{CO}$ molecules themselves (Ayres 1981), in what for all intents and purposes is 


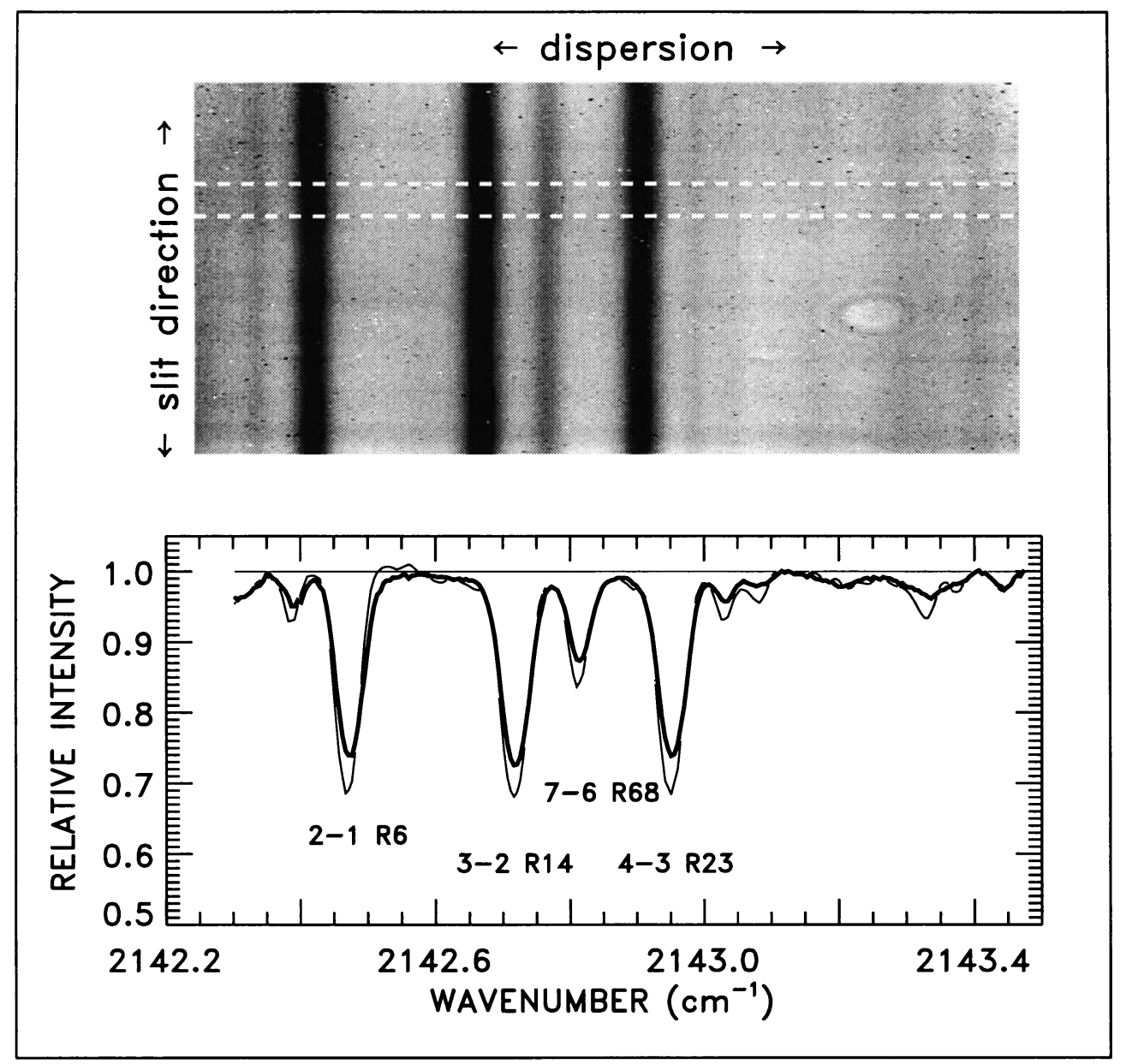

Figure 2. Long-slit double-pass spectrum from McMath-Pierce Main spectrograph.

merely an upward extension of the radiation-dominated photosphere.

I personally view the COmosphere as a relatively passive zone of the outer atmosphere, although some degree of mechanical heating can be tolerated before the low-temperature $\mathrm{CO}$-controlled equilibrium jumps to the high-temperature hot-line dominated branch (Ayres 1981; Anderson \& Athay 1989). The importance of the COmosphere comes in how one attributes the radiative activity of different levels of the atmosphere in a global assessment of the energy budget. The gas density is large in the 500-900 km range compared with the higher-lying canopy zone. Because the radiative cooling depends on $\sim n^{2}$, the intermediate altitude range is a key player in the energy budget. One 
can imagine that a calculation of total radiative losses (which must balance the input heating, and therefore are a proxy for it; see Vernazza, Avrett, \& Loeser 1981) will yield very different answers if there is a temperature rise in those layers (classical chromosphere) versus a temperature drop (COmosphere). If the thermal model is biased, gross errors in the inferred mechanical energy input are possible.

Now I will describe some of the evidence in favor of the COmosphere view.

\section{Observations of Solar CO in the Thermal Infrared}

In the late 1970s and early 1980s the instrument of choice for recording the solar $4.7 \mu \mathrm{m}$ CO fundamental bands was the $1 \mathrm{~m}$ Fourier transform spectrometer (FTS) on the McMath-Pierce telescope at Kitt Peak (Ayres \& Testerman 1981; Ayres, Testerman, \& Brault 1986; Ayres \& Brault 1990). Recent work, however, has turned to long-slit stigmatic imaging with the $13.5 \mathrm{~m}$ Main spectrograph, its newly-installed large $\left(419 \times 320 \mathrm{~mm}^{2}\right)$ IR grating, and a $256 \times 256$ Amber Engineering InSb camera commissioned in 1993 (see Uitenbroek, Noyes, \& Rabin 1994; Ayres \& Rabin 1996 [hereafter AR]). The all-reflecting unobscured design of the McMath-Pierce is vital in the thermal infrared, and the large (1.5 m diameter) primary provides diffraction-limited performance at $\sim 1^{\prime \prime}$, compatible with the best seeing routinely encountered at the telescope. The solar image can be stepped across the spectrograph slit to build up $2 \mathrm{D}$ surface maps, using a control system developed for NSO's Near Infrared Magnetograph (Rabin 1994).

Figure 2 illustrates a typical long-slit spectrum of the quiet Sun obtained at disk center on 9 May 1996. A tracing (thick curve, lower panel) of the portion highlighted by white dashed lines is compared with the NSO FTS photospheric atlas (Livingston \& Wallace 1991; thin curve, lower panel). During that run, we operated the spectrograph in double-pass mode. One obtains double the spectral resolution, at the expense of free spectral range. Oversampling the spectral image along both the dispersion and slit (spatial) directions renders the derived line parameters-central depth, width, and Doppler shift-less susceptible to flatfield errors. Double pass also suppresses, to some extent, the far wings of the instrumental profile responsible for scattered light, a particular nuisance in the grating spectrometer (and absent in the FTS). The scattering fills in the absorption cores of strong CO lines (Fig. 2), affecting the derived brightness temperatures (proxies for the kinetic temperatures at $\tau_{L} \sim 1$ ).

In classical scanning double-pass systems an intermediate slit clips the far profile wings, and a reading of diffuse light can be taken when the internal slit is closed. A narrow intermediate slit, however, would eliminate the spectral multiplex advantage. We therefore work around the scattered light by focussing on differential behavior (i.e., temperature changes), rather than ab- 


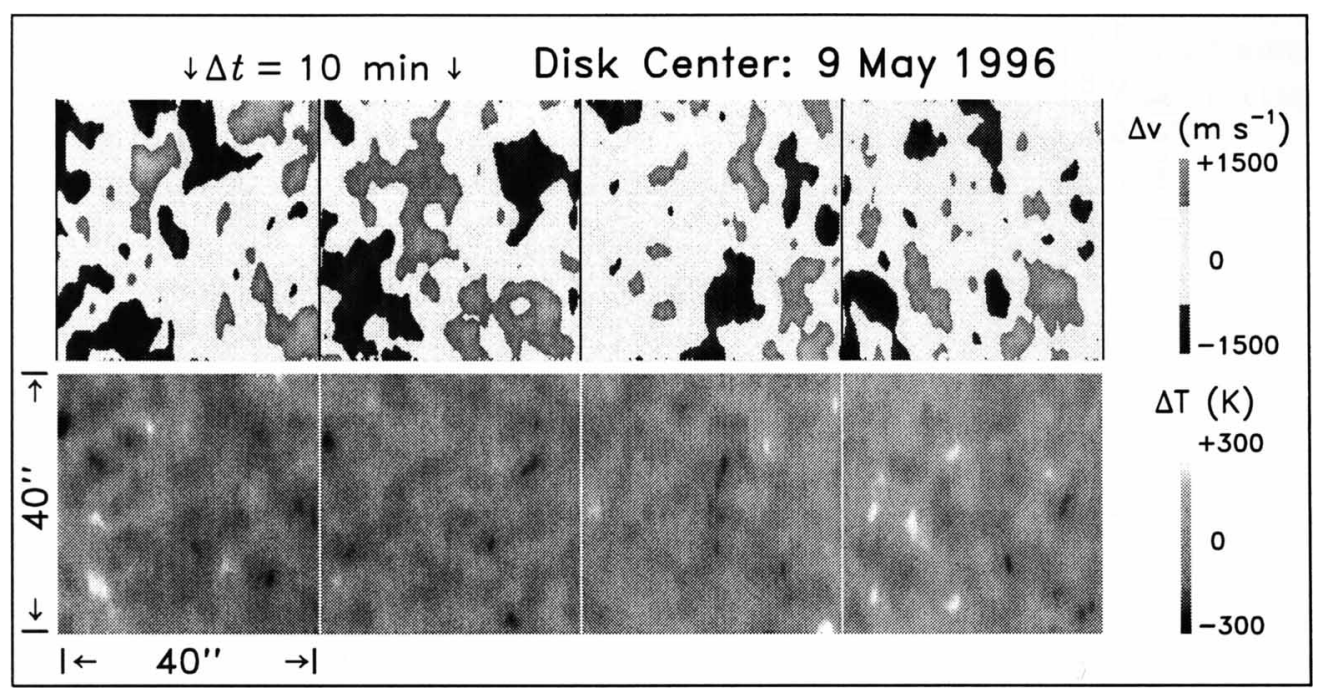

Figure 3. Velocity (upper) and temperature (lower) maps derived from strong CO lines.

solute quantities, and by incorporating a model of the scattering in our spectral simulations.

\subsection{THERMAL AND VELOCITY MAPS OF THE SOLAR SURFACE}

Figure 3 illustrates a sequence of four thermal and velocity maps derived from the three strong CO lines of the $2143 \mathrm{~cm}^{-1}$ interval shown in Fig. 2. The scans were obtained near disk center on 9 May 1996 under good observing conditions. Each frame is $40^{\prime \prime} \times 40^{\prime \prime}$ and required 10 minutes to complete. The color scales emphasize excursions beyond the normal fluctuations of the photospheric $p$ mode pattern at high altitudes (see AR). The velocity maps show $p$-mode interference "wavepackets." The thermal scenes are relatively bland, however, with no obvious very dark or very bright structures (say, with $|\Delta T|>500 \mathrm{~K}$ ), at least at the $\sim 2^{\prime \prime}-3^{\prime \prime}$ seeing-limited resolution of these frames. In larger-scale maps, one often can recognize persistent bright points coincident with $\mathrm{Ca} \mathrm{K}$ network fragments (e.g., AR), although none are evident in the sequence here.

\subsection{CO OFF-LIMB EMISSIONS}

The off-limb emissions of $\mathrm{CO}$ are a fundamental signature of cool gas in the altitude range corresponding to the low chromosphere of conventional models. $2 \mathrm{D}$ spherical transfer simulations suggest that molecular gas is present up to 


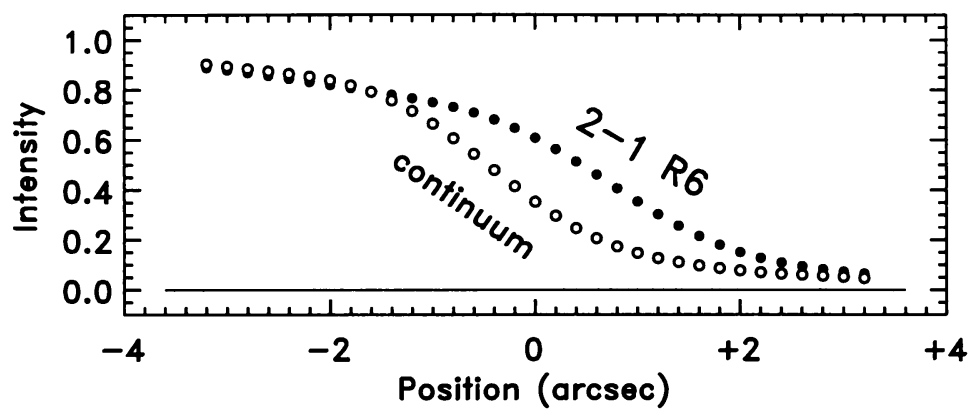

9 May 1996 coadd

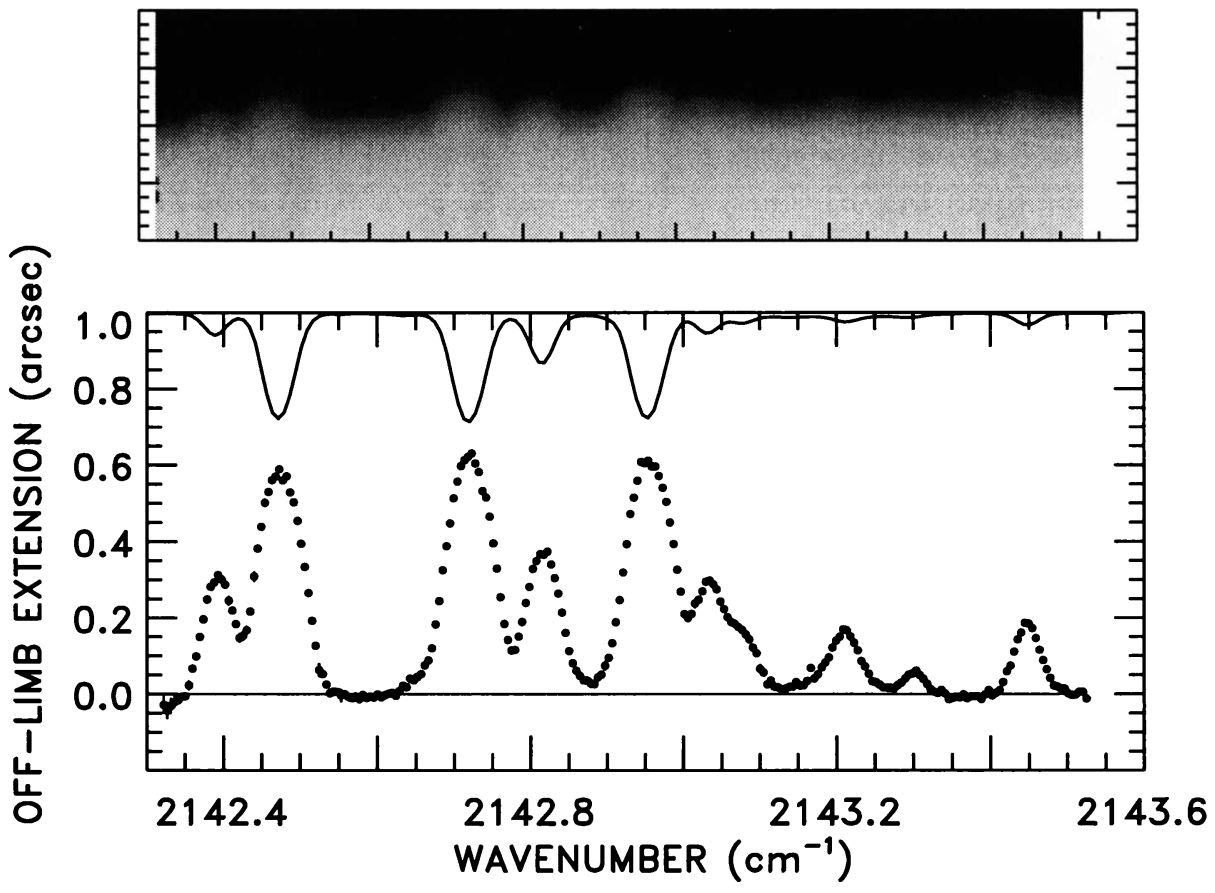

Figure 4. Off-limb emissions of CO lines in $2143 \mathrm{~cm}^{-1}$ region.

at least $900 \mathrm{~km}$. The emissions have a relatively low brightness temperature, $\sim 3500 \mathrm{~K}$, but are seen with high contrast against the dark sky (beyond the continuum edge). Figure 4 depicts the off-limb emissions in the $2143 \mathrm{~cm}^{-1}$ region. The double-pass observations were obtained during the morning of 9 May 1996 , under clear skies with moderately good seeing. The middle panel illustrates the coaddition of the 9 best spectrograms out of a run of 1000 , selected 
on the basis of limb sharpness (AR). The $\mathrm{CO}$ lines are the distinct mounds of emission extending beyond the bright continuum edge. The individual frames were registered by cross-correlation, and divided by an average on-disk spectral trace to normalize the intensities and suppress telluric absorptions.

The upper panel compares the translimb intensity profile of the $2142.6 \mathrm{~cm}^{-1}$ continuum with that of the core of the adjacent CO 2-1 R6 feature. The continuum edge should be sharp on a scale of $1^{\prime \prime}$; the apparent smoother roll-off is caused by seeing and diffraction. Nevertheless, the excess displacements of the $\mathrm{CO}$ emissions relative to the limb can be measured accurately as a differential effect. The lower trace in the bottom panel (dots) shows the spectrally-resolved off-limb extensions of the $2143 \mathrm{~cm}^{-1}$ interval relative to the continuum. The upper trace in the bottom panel (thin curve) illustrates a disk-center FTS relative intensity spectrum for comparison.

The strong $\mathrm{CO}$ absorptions at disk center show prominent extensions above the limb; the weaker features show larger extensions at the limb than one might anticipate from their relative strengths at disk center. The weaker lines have low opacities in the $500-900 \mathrm{~km}$ zone, but the tangential raypaths above the limb are tens of thousands of kilometers long, allowing the weak transitions to build up significant optical depths. On the other hand, the strong CO lines might achieve $\tau_{L}=1$-and thereby saturate-over only the first few hundred kilometers of the sightline.

\section{Interpretation}

\subsection{HOW PERVASIVE IS THE COOL GAS?}

The off-limb CO emissions are accumulated through an enormous tangential pathlength. They tell us directly that there is some cool gas present somewhere along the sightline, but not where or how much. The key question is how widespread the cool gas truly is.

To explain the extreme-limb darking of the $\mathrm{CO}$ line cores requires either: (1) cool material with a high filling factor (say, 80\%) at high altitudes; or (2) a smaller covering fraction (say, $\sim 20 \%$ ) of much colder structures. In the first case, the intensity behavior is dominated everywhere by the pervasive cool component. In the second case, the isolated cold, but transversely-opaque, "pillars" dominate only near the limb where they effectively "shadow" any hot structures (which naturally are transparent in $\mathrm{CO}$ ).

One consequence of the small filling factor model is that the cold regions (with $\Delta T \sim 1000 \mathrm{~K}$ ) should show up as small-scale "dark spots" with the $\sim 20 \%$ filling factor in disk-center thermal maps (AR). So far, within the sustained $\sim 2^{\prime \prime}-3^{\prime \prime}$ resolution typically encountered under good conditions at Kitt Peak, we have not seen anything remotely like the anticipated sprinkling of dark 


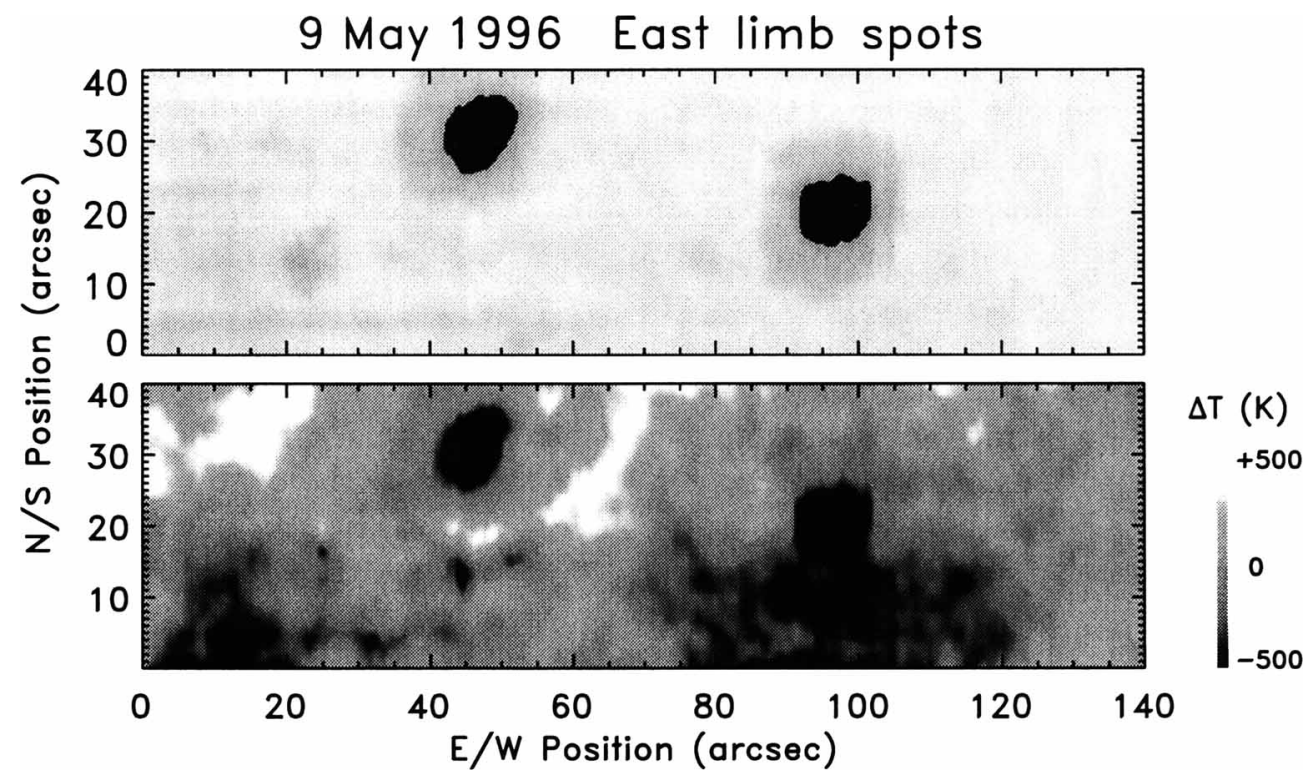

Figure 5. Long spatial scan through small spot group near east limb.

spots. We conclude that the opposite model—-pervasive COmosphere-is more promising.

We would feel more confident in our conclusion, of course, if we had access to subarcsecond spatial resolution (i.e., via a large-aperture all-reflecting solar telescope with active-optic image stabilization) to eliminate the (remote) possibility of ultra-fine-scale dark points, below the resolution of the present observations (see discussion in AR).

\subsection{DYNAMICAL COMOSPHERE?}

An alternative view of the COmosphere must be mentioned at this point (Carlsson \& Stein 1995; Avrett et al. 1996). 1 D radiation-hydrodynamics simulations suggest that the $500-1000 \mathrm{~km}$ altitude range might be in a continually evolving thermal state owing to the passage of steepening acoustic wavetrains from below. At those low densities, the $\mathrm{CO}$ chemical timescales are long (see $\mathrm{AR}$ ). The molecular population cannot respond to the instantaneous temperature perturbations, but rather attains an equilibrium based on the timeaverage thermal profile (rather cool in the Carlsson \& Stein model). Such a steady-state background population of $\mathrm{CO}$, existing in a fluctuating thermal environment, might be capable of explaining the off-limb emissions and 
extreme-limb darkening of the infrared $\Delta v=1$ lines. At the same time one would obtain significant time-averaged ultraviolet signatures from the same spatial volume (mostly from the extreme temperature peaks). The observational consequences should be pursued further; as well as the incorporation of the CO chemistry (and cooling) into the dynamical simulations.

\subsection{THERMAL SHADOWS IN ACTIVE REGIONS?}

What I have presented is mostly in the way of review. I will conclude with something new, a brief discussion of an intriguing observation of the $\mathrm{CO}$ lines in an active region.

Figure 5 displays a thermal scan across a small spot group, near the east limb, which D. Rabin and I followed for four days of our observing run in early May 1996 . The upper panel shows a $2142.6 \mathrm{~cm}^{-1}$ continuum map. We see the two dark sunspots and, faintly, the surrounding penumbral regions. In the lower panel, the $\mathrm{CO}$ thermal map again displays the cold umbrae, although at the heights of the photosphere probed by the $\Delta v=1$ lines, the penumbrae show little if any contrast. We also see bright plage regions, which have no counterpart in the continuum images. The warm, low-density flux tubes that comprise the plages act as low-opacity windows through the molecular zone of the high photosphere, revealing the hot continuum below.

The fact that sunspots are dark in $\mathrm{CO}$, and plages are bright is not new. What is different in these maps are the regions of $\sim 500 \mathrm{~K}$ cooler temperatures to the south of the westward spot, and to the south east of the eastward spot. These areas are much cooler than anything seen in the quiet Sun (e.g., Fig. 3), but are not connected with any obvious spot-like structures in the continuum map. Possibly, they are associated with near-surface horizontal fields which partially suppress convection and thereby steepen the temperature gradient (yielding cooler temperatures higher up, since the absolute temperature $T$ near $\tau_{C} \sim 1$ is pegged by the constraint to radiate away the energy flux from below: $\left.\mathcal{F} \sim T^{4}\right)$.

Such "thermal shadows" might provide insight into the subsurface character of active regions, and possibly allow one to anticipate the future evolution of a growing or decaying spot group; relevant to predicting solar activity as well as to understanding the dynamo at its heart (Parker 1970).

I offer this preliminary untested result as an indication of how much we have yet to learn in the exciting rapidly-developing arena of infrared solar physics. 
Acknowledgments. I thank D. Rabin and D. Jaksha for their help with the project. This work was supported by grant AST-9618505 from the National Science Foundation to the University of Colorado. I also thank the International Astronomical Union for a partial travel grant to the Kyoto General Assembly (and this Symposium).

\section{References}

Anderson, L. S., \& Athay, R. G. 1989, ApJ, 346, 1010

Athay, R. G. 1976, The Solar Chromosphere and Corona: Quiet Sun, (Dordrecht: D. Reidel) Avrett, E. H. 1997, in Synoptic Solar Physics, 18th NSO/Sacramento Peak Summer Workshop, ed. K. Balasubramaniam, J. Harvey, \& D. Rabin, (Tucson: NSO), in press

Avrett, E. H., Höflich, Uitenbroek, H., \& Ulmschneider, P. 1996, in Cool Stars, Stellar Systems, and the Sun, 9th Cambridge Workshop, ed. R. Pallavicini \& A. K. Dupree, ASP Conf. Ser., Vol. 109, p. 105

Ayres, T. R. 1979, ApJ, 228, 509 1981, ApJ, 244, 1064 1997, in Synoptic Solar Physics, 18th NSO/Sacramento Peak Summer Workshop, ed. K. Balasubramaniam, J. Harvey, \& D. Rabin, (Tucson: NSO), in press

Ayres, T. R., \& Brault, J. W. 1990, ApJ, 363, 705

Ayres, T. R., \& Rabin, D. 1996, ApJ, 460, 1042 (AR)

Ayres, T. R., \& Testerman, L. 1981,

Ayres, T. R., Testerman, L., \& Brault, J. W. 1986, ApJ, 304, 542

Carlsson, M., Judge, P. G., \& Wilhelm, K. 1997, ApJ, 486, L63

Carlsson, M., \& Stein, R. F. 1995, ApJ, 440, L29 1997, ApJ, 481, 500

Dere, K. P., Bartoe, J.-D. F., \& Brueckner, G. E. 1989, Solar Phys., 123, 41

Fontenla, J. M., Avrett, E. H., \& Loeser, R. 1993, ApJ, 406, 319

Foukal, P. V. 1975, Solar Phys., 43, 327

Hirayama, T. 1985, Solar Phys., 100, 415

Innes, D. E., Inhester, B., Axford, W. I., \& Wilhelm, K. 1997, Nature, 386, 811

Gurman, J. B. 1997, in Cool Stars, Stellar Systems, and the Sun, 10th Cambridge Workshop, ed. R. A. Donahue \& J. A. Bookbinder, ASP Conf. Ser., in press

Livingston, W., \& Wallace, L. 1991, An Atlas of the Solar Spectrum in the Infrared from 1850 to $9000 \mathrm{~cm}^{-1}(1.1$ to $5.4 \mu \mathrm{m})$, NSO Tech. Rept. No. 91-001

Moses, D., et al. 1994, ApJ, 430, 913

Noyes, R. W., \& Hall, D. N. B. 1972, BAAS, 4, 389

Parker, E. N. 1970, ARA\&A, 8, 1 1983, ApJ, 264, 635

Rabin, D. M. 1994, in IAU Symp. 154, Infrared Solar Physics, ed. D. M. Rabin, J. T. Jefferies, \& C. Lindsey (Dordrecht: Kluwer), 449

Sheeley, N. R. 1969, Solar Phys., 9, 347

Solanki, S. K., Livingston, W., \& Ayres, T. 1994, Science, 263, 64

Solanki, S. K., \& Steiner, O. 1990, A\&A, 234, 519

Suematsu, Y., Wang, H., \& Zirin, H. 1995, ApJ, 450, 411

Uitenbroek, H., Noyes, R. W., \& Rabin, D. 1994, ApJ, 432, L67

Vaiana, G. S., \& Rosner, R. 1978, ARA\&A, 16, 393

Vernazza, J. E., Avrett, E. H., and Loeser, R. 1973, ApJ, 184, 605 1981, ApJS, 45, 635

Wiedemann, G., Ayres, T. R., Jennings, D. E., \& Saar, S. H. 1994, ApJ, 423, 806

Zirin, H. 1988, Astrophysics of the Sun, (Cambridge: Cambridge Univ. Press) 